\title{
Laser-phakopuncture in the treatment of soft cataracts
}

\author{
M. M. KRASNOV \\ State Institute of Ophthalmology, Moscow, U.S.S.R.
}

The use of lasers in medicine is a fairly recent development. Clinical studies have usually made use of the thermal (coagulating) effect of laser irradiation. The application of lasers in ophthalmology has so far been confined mainly to lesions of the fundus, such as retinal detachment and diabetic retinopathy. Other (non-thermal) effects have more recently been investigated and have been employed for the first time in clinical ophthalmology (Krasnov, 1972).

In contemporary surgery, published papers show that there is interest in the possibility of using a laser beam instead of a knife. This is seen in neurosurgery, dermatology, and otolaryngology. In biology the same applies (laser microsurgery of the cell).

The transparency of the ocular media makes the use of lasers especially promising. In some diseases the thermal action is exactly what is needed to serve the aim of the treatment, for example to achieve coagulation of the newly formed blood vessels in diabetic retinopathy. For many other purposes, however, the burn to the ocular structures is contraindicated, and it is the mechanical, cutting effect that is required. An interesting way of solving this problem is the use of Q-switched (or modulated) pulses of laser energy. Their main characteristic is their extremely short duration of only a few nanoor picaseconds ( $\mathrm{IO}^{-8} \mathrm{~s}$ and less).

We started to work along these lines, having consulted the Nobel laureate, A. M. Prokhorov, who confirmed the theoretical validity of the underlying idea.

The anterior segment of the eye presents considerable scope for the use of the laser for purposes previously achieved by surgery: this "laser microsurgery" of the anterior segment of the eye is virtually a new field of exploration.

Since 1970 the author has had much experience in the laser treatment of glaucoma. The procedure of Q-switched laser goniopuncture, laser iridectomy, and laser cyclodialysis have been advanced and developed for clinical use in different forms of glaucoma (Krasnov, 1973; Krasnov, Saprykin, Doronin, Nikolskaya, Akopyan, and Mamedov, 1973). The laser was also successfully employed to fix an intraocular lens to the iris (extrapupillary iris-lens: Krasnov and Orlova, 1969).

Address for reprints: M. M. Krasnov, M.D., State Institute of Ophthalmology, Moscow 5 Pogodinskaya St., U.S.S.R.
In the course of this work a number of basic problems had to be solved concerning the principles and effects inherent in the interaction of a laser beam with the ocular tissue at the target point. Among them were the study of the optimum characteristics of the laser impulses (duration, output, energy, etc.) in different clinical situations, and of channelling powerful laser beams into the eye, dosage, safety requirements, etc. Experimental studies on animal eyes were carried out in preparation for the clinical work.

A new Q-switched laser unit was designed (Krasnov, 1972), mainly for the use in the anterior segment of the eye. The present communication is confined to the subject of cataract treatment.

The laser has already been successfully tried for the perforation of the secondary membranes in the pupillary region (Eroshevsky, Panfilov, and Semenov, 1971; Krasnov and others, I973; Massin and Gernet, 1973), but no reports have been traced by the author on the use of the laser in non-membranous cataracts.

The basic idea of the present investigation stemmed from data obtained from some of our studies, which seemed to support the possibility of breaking the lens capsule by a Q-switched laser pulse. The application of such a method is, by its very principle, limited to so-called "soft" cataracts, in which a conventional discission procedure might have been the method of choice. It should be pointed out that the procedure of discission once held a prominent place in the surgery of soft cataracts, but that the operation later lost much of its popularity, mainly because it involved the risk of complications such as phakoanaphylactic uveitis and phakolytic glaucoma. The availability of treatment to control these complications has greatly increased since that time.

The above considerations constituted a basis for the experimental treatment of cataract with the laser.

First, experiments were carried out on the eyes of thirty chinchilla rabbits to investigate the foundations of the method. Q-switched ruby and neodymium lasers were used with the output energy of a single pulse (measured at the target point) of up to $0.3 \mathrm{~J}$ and a pulse duration of about 20 nanoseconds. It was easy to demonstrate the rupture of the lens capsule (as a rule, with the first pulse).

Strong pulses of laser energy may be harmful to the 
inner structures of the eye: this particularly concerns the neodymium lasers. Certain precautions are therefore needed to prevent retinal damage, one of the most important being very short focusing.

Our experimental data will be summarized in a separate communication. The main conclusion was the feasibility of clinical trials with human subjects.

From 1972 onwards, the procedure of $Q$-switched laser phakopuncture was tried on human eyes. In the first place ten eyes with senile cataract were treated, and in none was the capsule ruptured. This revealed the important fact that the anterior capsule of the human lens is more resistant to laser irradiation than that of the rabbit lens. Eventually all the eyes thus treated were subjected to a conventional cataract extraction; it was found that there had been no damage to the visual function, and the procedure was then tried for the treatment of soft cataract.

Laser phakopuncture was carried out on the eyes of a series of nine children, whose ages ranged from 4 to 12 years. The cataract was congenital in six cases and traumatic in two, and in one the underlying cause remained uncertain (? uveitis). Only topical anaesthesia (for instance, I-2 drops of $0.5^{-1}$ per cent. pontocaine) was required.

As with the adults (see above) the capsule could not be ruptured as easily as in our experiments on animal eyes. More sophisticated ways had therefore to be found. Nature sometimes affords an easy solution whenever there are dark (pigmented) deposits on the capsule: these are potential absorbents of light energy, and the capsule is, as a rule, easily torn at such points by an incident laser pulse. If the capsule is transparent throughout its entire surface, it is sometimes possible to puncture it by delivering the pulse at the pupillary border. The pigment granules accumulated in this region serve as photoacceptors, but they, naturally, must be in contact with the capsule. Some injury to the pupillary margin is thus unavoidable, but there is virtually no harm in this. The lesion can, of course, be conveniently localized under the upper lid.

In another method which proved to be reliable, the procedure was divided into two stages:

(I) The iris was pre-treated with the laser in a similar way to that used in cases of retinal detachment, i.e. by producing a burn in the iris tissue which caused an iridocapsular adhesion. The posterior synechiae thus formed are always pigmented and present convenient target-points for a subsequent laser phakopuncture.

A conventional argon laser can be employed for this pretreatment procedure. The pupil should be made conveniently narrow by the use of a suitable miotic, and should be kept constricted for several days afterwards to enhance the formation of the synechiae.
(2) For the second stage, on the contrary, full mydriasis is required. The posterior synechiae are either ruptured or become exposed, thus providing the pigmented target-points of light absorption. Conventional (non-modulated) lasers can sometimes be employed for this second-stage procedure also).

With the use of the techniques described, laser capsulotomy was eventually produced in all our cases. Mydriasis needs to be maintained continuously for a period of several months afterwards to prevent the puncture site from becoming closed by the iris.

A successful Q-switched laser-phakopuncture starts a slow process of liquefaction and resorption of the lens substance. As a rule, there is no massive herniation of the lens material into the anterior chamber because the hole in the capsule is too small. On the contrary, we obseryed a tendency to gradual obliteration of the defect by proliferation of the capsular epithelium. Closure of the capsular rupture is an indication to repeat the laser "incision".

Complete reabsorption of the lens substance in our patients took several months (up to approximately one year). Signs of a very mild inflammatory response could sometimes be detected, but subsided quickly after local treatment with steroid drops. There were no other complications.

The most important result of our investigations is the possibility of achieving aphakia without surgery. Q-switched laser-phakopuncture should of course never be attempted in the case of cataracts with a hard nucleus or with signs of tissue proliferation (for instance, after long-standing uveal inflammation).

The anatomical results in our cases were, on the whole, better than the functional ones. In spite of complete reabsorption of the lens substance, full visual acuity was achieved in only two cases out of nine, although there were no clinical signs of retinal damage due to laser irradiation. It therefore seems probable that some of the eyes had been amblyopic; this is often the case with congenital cataracts, and is often responsible for the poor visual results of surgical treatment.

\section{Summary}

A new method of treating soft cataracts by laser irradiation is described ("laser-phakopuncture"). Rupture of the lens capsule can be produced by a strong laser pulse focused on the anterior surface of the lens. This is followed by gradual reabsorption of the lens substance (in much the same manner as with a conventional discission procedure). Pigment deposits on the capsule are usually necessary to ensure sufficient absorption of light energy at the target point. The laser beam can also be employed in a preliminary procedure to produce such pigmented spots, and to prepare the eye for a subsequent laserphakopuncture. 
The method was first investigated experimentally on animal eyes. The so-called Q-switched lasers proved to be most effective in producing a tear in the capsule. It was then tried clinically in nine juvenile patients and reabsorption of the lens substance was the eventual result in all cases, although full visual acuity was achieved in only two of them.

The use of lasers offers a completely new approach to the treatment of cataract without surgery.

\section{References}

ERosheVsky, T. I., PANFilov, N. J., and Semenov, A. D. (1971) Vestn. Ophthal., No. 2, p. Io KRASNOV, M. M. (1972) Ibid., No. 3, p. 27 (1973) Amer. F. Ophthal., 75, 674 and ORLova, E. M. (1969) Vestn. Ophthal., No. 3, p. 47 , SAPRYKin, P. T., DORONin, P. P., NIKOlSKayA, G. M., AKOPYAN, v. S., and MAMEdov, N. G. (I973)

Ibid., No. 2, p. 9

massin, m., and gernet, H. (1973) Klin. Mbl. Augenheilk., 162, 369 